Revista Eletrônica de Ciência Administrativa (RECADM) - ISSN 1677-7387

Faculdade Cenecista de Campo Largo - Coordenação do Curso de Administração

v. 3, n. 1, maio/2004 - http://revistas.facecla.com.br/index.php/recadm/

\title{
ROTATIVIDADE DOS EXECUTIVOS E GOVERNANÇA CORPORATIVA NO BRASIL
}

\author{
Charles De Montreuil Carmona, Doutor \\ Departamento de Ciências Administrativas/PROPAD/UFPE \\ Rua Prof. Moraes Rego, 1235 - Cidade Universitária, Recife, PE - CEP:50670-901 \\ e-mail: carmona@ufpe.br \\ Fones: (81) 3271-8368/3274-7176 \\ R.168 Fax: (81) 3271-8870
}

\section{Resumo}

Este trabalho avalia as determinantes da rotatividade executiva e a valorização da firma em função da propriedade e estrutura de controle no mercado brasileiro, o qual apresenta baixa proteção para os investidores e empresas com poucos acionistas controladores. Também analisa a sensibilidade dessa rotatividade em relação ao tamanho da empresa, rentabilidade das ações e o índices $Q$ de Tobin. As hipóteses testadas neste trabalho analisam a relação entre a rotatividade executiva e os conflitos de interesses potenciais entre controladores e administradores. Um conjunto de 46 empresas com ações negociadas na Bolsa de Valores de São Paulo (BOVESPA) foi analisado durante o período de 19972000, utilizando o modelo Probit, e foram encontrados resultados estatisticamente significantes em relação à causalidade da variável tamanho das empresas em relação ao nível de rotatividade dos executivos. Não foram obtidos resultados conclusivos em relação à variável que incorpora a mudança na estrutura de propriedade.

Palavras-chave: Eficiência Financeira, Governança Corporativa, Modelos Probit.

\section{Top executive turnover and Corporate Governance in Brazil}

\begin{abstract}
This work evaluates top executive turnover in function of the property and structure of control in the Brazilian market, which presents low protection for the investors and companies. Also it analyzes the sensitivity of this rotation in relation to the size of the company, yield of the actions and indices $Q$ of Tobin. The hypotheses tested in this work analyze the relation between the executive rotation and the conflicts of potential interests between controllers and administrators. A set of 46 companies listed in the Stock exchange of São Paulo was analyzed during the period of 1997-2000, using the Probit model, It was founded results estatistically significants in relation to the causalidade of the companies in relation to top executives turnover.
\end{abstract}

\section{Introdução}


Revista Eletrônica de Ciência Administrativa (RECADM) - ISSN 1677-7387

Faculdade Cenecista de Campo Largo - Coordenação do Curso de Administração v. 3, n. 1, maio/2004 - http://revistas.facecla.com.br/index.php/recadm/

Atualmente existe um crescente interesse por examinar mecanismos de proteção de investidores e instituições do mercado financeiro por meio de sistemas de controle eficientes. A questão relevante consiste em verificar que o controle dos ativos seja realizado pelos agentes melhor qualificados para poder agregar valor a esses ativos. Este assunto pode chegar a ser crucial em países como o Brasil, porque a ineficiência dos sistemas de governança corporativa das empresas é um fator limitante do crescimento dos negócios, inibindo a capacidade de captação de recursos.

A literatura financeira existente indica duas estratégias para avaliar a eficiência do sistema de governança corporativa das empresas. A primeira, segundo Kaplan (1994) e Coffee (1999) seria testar se o aumento na rotatividade dos principais executivos de uma empresa é resultado do desempenho declinante da organização. Assim, Coffee conclui que os sistemas de governança bem sucedidos punem os executivos quando existe um baixo desempenho dos preços das ações da empresa ou apresenta níveis reduzidos de fluxos de caixa. Estudos confirmando esses resultados para diversos países foram também realizados por Warner, Watts e Wruck (1988), Franks e Mayer (2000), entre outros.

Uma segunda estratégia, derivada de Morck, Shleifer e Vishny (1988) e McConnell e Servaes (1990), resulta de analisar o valor da firma em relação a companhias similares.

As duas estratégias mencionadas mensuram a qualidade do padrão de governança de uma empresa. Complementarmente a esses trabalhos, La Porta, Lopez-de-Silanes e Shleifer (1999) e Bebchuk (1999) analisaram a alta concentração da propriedade em países de baixa proteção aos investidores, nesses casos, os controladores têm os incentivos e o poder para disciplinar as decisões gerenciais. Assim, criam-se as condições de rotatividade de executivos e de prováveis conflitos de interesses com os acionistas minoritários.

Volpin (2001) estabelece uma análise integrada para avaliar a eficiência do sistema de governança corporativa considerando em profundidade as principais variáveis determinantes da elevada rotatividade dos executivos nas empresas e também incorporando a mudança no valor das empresas, ou seja, as duas estratégias anteriores. Volpin examina a estrutura de propriedade e controle de uma amostra de empresas italianas, selecionando os elementos determinantes de um mercado, que ao igual que a estrutura brasileira, se caracteriza por poucos acionistas controladores e pouca proteção legal para os investidores.

Este trabalho estudará primeiro as determinantes da rotatividade executiva em companhias brasileiras, para focalizar, em particular, como a propriedade e a estrutura de controle da firma afeta a sensibilidade da rotatividade executiva considerando resultados históricos das empresas. Então, avalia-se os efeitos destes mesmos fatores sobre $o$ índice $Q$ de Tobin das firmas, assim como $o$ 


\section{Revista Eletrônica de Ciência Administrativa (RECADM) - ISSN 1677-7387 \\ Faculdade Cenecista de Campo Largo - Coordenação do Curso de Administração \\ v. 3, n. 1, maio/2004 - http://revistas.facecla.com.br/index.php/recadm/}

tamanho e o resultado operacional das mesmas. Este estudo baseia-se em um amplo conjunto de dados, os quais abrange empresas industriais, serviços e instituições financeiras durante o período de 1997-2001 e contendo informações sobre propriedade, estrutura do Conselho de administração e do capital, entre outras informações.

\section{Desenvolvimento}

O tema relativo ao conflito de interesses entre administradores e proprietários é importante dentro do estudo da governança corporativa. Em geral, o interesse relativo às partes envolvidas dentro das relações organizacionais é conhecido como teoria de agência. De acordo com Jensen e Meckling (1996), o problema geral de agência é o de induzir os agentes a agirem com o objetivo de maximizar os interesses dos proprietários. Porém, essa indução envolve delegação de decisões, sendo que essa faculdade de decidir pode ser motivada por desejos de status, poder, renda e estabilidade (Hill e Jones, 1998).

Em primeiro lugar, devem ser discutidos os aspectos substanciais a toda efetiva governança corporativa.

\subsection{Governança Corporativa e seu impacto esperado nas empresas}

A Governança Corporativa aborda aspectos relacionados ao Conselho de Administração, como Proprietários, Auditoria Independente, Conselho Fiscal, Transparência, o Justo Peso dos Poderes, Prestação de Contas, Ética e Conflito de interesse, entre outros.

O objetivo central das Práticas de Governança Corporativa é indicar caminhos para todos os tipos de empresas: sociedades por ações de capital aberto ou fechado, limitadas ou sociedades civis, visando melhorar o desempenho das empresas e facilitar o acesso ao capital.

As principais linhas estabelecidas são três: Transparência (Disclosure), Prestação de Contas (accountability) e, Eqüidade. Dessa maneira, atua na redução do risco e aumento do valor da empresa por proporcionar uma melhor gestão, maior transparência, melhor avaliação da organização, e permitir a discussão da estratégia entre o conselho de administração e os executivos da empresa. Também permite a avaliação da qualidade da administração, a maior confiança por parte dos stakeholders e proteção aos acionistas minoritários (Documento técnico da OECD, 1999).

Os investidores externos consideram que a adoção dessas práticas valorizam o retorno do investimento dos acionistas e do valor da empresa. Assim, conduzem à redução dos riscos da tomada de decisões ou de adoção de táticas empresariais erradas. 
Revista Eletrônica de Ciência Administrativa (RECADM) - ISSN 1677-7387

Faculdade Cenecista de Campo Largo - Coordenação do Curso de Administração

v. 3, n. 1, maio/2004 - http://revistas.facecla.com.br/index.php/recadm/

\subsection{Variáveis de referência dos modelos de avaliação de sistemas de Governança Corporativa}

Estudos analisando as relações de dependência que existem entre a rotatividade da diretoria e diferentes variáveis de desempenho empresarial, incluindo a mudança da participação acionária, apresentam modelos que incorporam muitas variáveis causais ou regressores. Por exemplo, Volpi (Op. Cit.), examina um modelo de 20 variáveis independentes causais da rotatividade empresarial, dentre as quais encontram-se: os direitos de fluxos de caixa dos controladores, percentual de acionistas minoritários, níveis piramidais, percentual de altos executivos que foram substituídos, altos executivos que são parentes dos controladores, incentivos de remuneração, índice de valor $Q$, entre outras. Gompers (2001) também apresenta um modelo multivariado com intuito de construir um índice de governança.

Neste trabalho, de acordo com as características do mercado financeiro brasileiro e as possibilidades de obtenção de dados confiáveis, podemos considerar como variáveis relevantes:

a) Mudanças no Lucro Operacional $\left(\Delta L O_{i t}\right)$. Incorpora a variação do lucro operacional (LO) padronizado através da média dos ativos totais ( $A T)$,

$$
\Delta L O_{i t}=\left(L O_{i t}-L O_{i t-1}\right) /\left(0,5 * A T_{i t}+0,5 * A T_{i t-1}\right)
$$

Onde a mudança na variável operacional mensura a eficiência operacional através capacidade dos ativos totais na geração de lucro, visualizando assim o clássico paradigma da teoria financeira de maximização da riqueza dos proprietários.

b) Tamanho $\left(T_{i t}\right)$. É uma variável da escala dos ativos totais das empresas analisadas. Pode ser calculada aplicando a função logaritmo à média dos ativos totais em dois períodos consecutivos

$$
T_{i t}=\operatorname{Ln}\left[0,5 * A T_{i t}+0,5 * A T_{i t-1}\right]
$$

Quanto maior o tamanho do negócio tanto maior a possibilidade de mudança da administração que, em alguns casos, resulta de um processo de aquisição ou fusão.

c) Retorno das ações da empresa (R). Calculado de acordo com as variações nos preços das ações $\left(P_{i}\right)$ entre o período t e t-1 e também inclui a taxa obtida pela distribuição de dividendos,

$$
R_{i t}=\frac{\left(P_{i t}-P_{i t-1}\right)}{\left(0,5 * P_{i t}+0,5 * P_{i t-1}\right)}+T \cdot \text { Div }_{\cdot i t}
$$




\section{Revista Eletrônica de Ciência Administrativa (RECADM) - ISSN 1677-7387 \\ Faculdade Cenecista de Campo Largo - Coordenação do Curso de Administração \\ v. 3, n. 1, maio/2004 - http://revistas.facecla.com.br/index.php/recadm/}

Dessa maneira, a variável $R_{i}$ relaciona os resultados em função do valor de mercado das ações, ou seja, através da percepção do mercado perante as prováveis condições de crescimento do negócio.

d) Índice $\mathbf{Q}$ de Tobin. Como foi descrito anteriormente, calcula-se mediante a soma do Capital próprio da empresa mais os passivos de longo prazo, dividido pelos Ativos totais da empresa. Este indicador relaciona o valor de mercado da soma de endividamento e do capital próprio de uma empresa, por outro lado, dividido pelo custo de reposição de seus ativos.

Assim, este indicador é semelhante ao do valor de mercado relacionado com o valor contábil, mas existem algumas pequenas diferenças. O numerador de $Q$ inclui todos os títulos de dívida e o capital próprio da empresa, e não apenas as ações ordinárias. O denominador inclui todos os ativos e não apenas o valor líquido da empresa.

De forma geral, as empresas têm um incentivo para investir quando o $Q$ é superior a 1 , indicando que existem boas probabilidades de crescimento. Também deve esperar-se que essas empresas mantenham uma grande vantagem competitiva em relação a outras de $Q$ baixo.

e) Mudança na Participação Acionária (PA). Variável dummy que toma o valor 1 se muda o controle acionário entre o período t e $\mathrm{t}+1$ e o valor 0 , caso contrário.

f) Rotatividade dos executivos $(\boldsymbol{y})$. Representa a variável dependente. É estruturada como uma variável probabilística e dicotômica que representa a alteração no cargo de algum dos altos executivos, apresentando o valor 1 em caso de mudança de executivos e o valor nulo, caso contrário.

\subsection{Modelos Probit}

O modelo Probit de probabilidade está associado à função cumulativa de probabilidade normal. Nosso interesse primário consiste em interpretar a variável dependente do modelo como a probabilidade de fazer uma escolha dada informações sobre os atributos individuais. $\mathrm{O}$ requerimento de tal processo é que traduz o valor do atributo, o qual pode oscilar no valor sobre a inteira linha real, para uma probabilidade que pode variar do valor 0 para 1. Para compreender este modelo, assumese que existe um índice teórico Zi (índice estimado), que é determinado por uma variável explanatória $X \mathrm{i}$, como no modelo linear de probabilidade. O índice $\mathrm{Zi}$ é suposto ser uma variável aleatória contínua e normalmente distribuída por razões econométricas usuais. Daí, podemos escrever:

$$
\mathrm{Zi}=\alpha+\beta \mathrm{X} i
$$


Revista Eletrônica de Ciência Administrativa (RECADM) - ISSN 1677-7387

Faculdade Cenecista de Campo Largo - Coordenação do Curso de Administração

v. 3, n. 1, maio/2004 - http://revistas.facecla.com.br/index.php/recadm/

O problema na análise probit é como obter estimativas para os parâmetros $\alpha$ e $\beta$ enquanto que ao mesmo tempo obtêm informações sobre a escala básica do índice $z$ não mensurada.

Uma fórmula alternativa é assumir um modelo de regressão:

$$
\mathrm{Y}_{i}^{*}=\beta_{0}+\sum_{j=1}^{k} \beta_{i} \mathrm{X}_{i j}+u_{i}
$$

Onde $Y^{*}$ é não observável, chamada comumente de uma variável latente. O que na prática observamos é uma variável dummy yi definida por

$$
\begin{array}{ll}
y_{i}=1 & \text { se } y^{*}>0 \\
y_{i}=0 & \text { se } y^{*}<0
\end{array}
$$

A diferença entre o modelo Probit e o modelo de regressão linear, é que no primeiro analisamos as variáveis dicotômicas como são, enquanto que no segundo assumimos a existência de uma variável fundamental latente para as quais observamos uma realização bifurcada.

$$
\left.P_{i}=\operatorname{Pr} o b\left(\mathrm{Y}_{i}=1\right)=\operatorname{Pr} o b\left[u_{i}\right\rangle-\left(\beta_{0}+\sum_{J=1}^{K} \beta_{i} \mathrm{X}_{i j}\right)\right]=1-F\left[-\left(\beta_{0}+\sum_{J=1}^{K} \beta_{I} \mathrm{X}_{I J}\right)\right]
$$

Onde F é a distribuição cumulativa de $u$.

Se a distribuição de $u$ é simétrica, desde que $1-F(-Z)=F(Z)$, podemos escrever,

$$
P_{I}=F\left(\beta_{0}+\sum_{j=1}^{K} \beta_{i} X_{i j}\right)
$$

O modelo Probit assume que $Z_{i}^{*}$ é uma variável aleatória normalmente distribuída, então $Z_{i}^{*}$ é menor (ou igual) a $Z_{i}$, podendo ser computada como uma função de probabilidade cumulativa normal (esta função assume para um número $Z$ a probabilidade de que qualquer $Z^{*}$ arbitrário será menor ou igual a Z).

A variável Pi estará no intervalo $(0,1)$. Pi representa a probabilidade de um evento ocorrer. Esta probabilidade é mensurada num intervalo abaixo da curva normal de $-\infty$ a $Z_{i}^{*}$, o evento será mais provável ocorrer além do valor do índice $Z_{i}$. 


\section{Revista Eletrônica de Ciência Administrativa (RECADM) - ISSN 1677-7387 \\ Faculdade Cenecista de Campo Largo - Coordenação do Curso de Administração \\ v. 3, n. 1, maio/2004 - http://revistas.facecla.com.br/index.php/recadm/}

Para obter uma estimativa do índice $Z_{i}$, aplicamos o inverso da função cumulativa normal:

$$
\mathrm{Z}_{i}=F\left(P_{i}\right)=\alpha+\beta \mathrm{X}_{i}
$$

Podemos interpretar a probabilidade Pi resultante do modelo Probit como uma estimativa da probabilidade condicional que um voto positivo individual, dado que o resultado individual é $X \mathbf{i}$. Isto é equivalente à probabilidade que uma variável normal padrão será menor que ou igual a $\alpha+\beta X$.

Observe-se que o declive de uma função Probit como mostra a figura 1 é mais amplo que a função de probabilidade linear no meio do espaço entre os valores extremos. O gráfico sugere algumas dificuldades que podem ser associadas à incorreta especificação do modelo de probabilidade linear. Assume-se, por exemplo, que a especificação Probit é correta. A estimativa do modelo linear incorreto conduzirá à falsa conclusão que o declive da função é constante quando de fato a mudança na probabilidade associada com as mudanças em $X$ é dependente sobre o valor particular de $X$ selecionado.

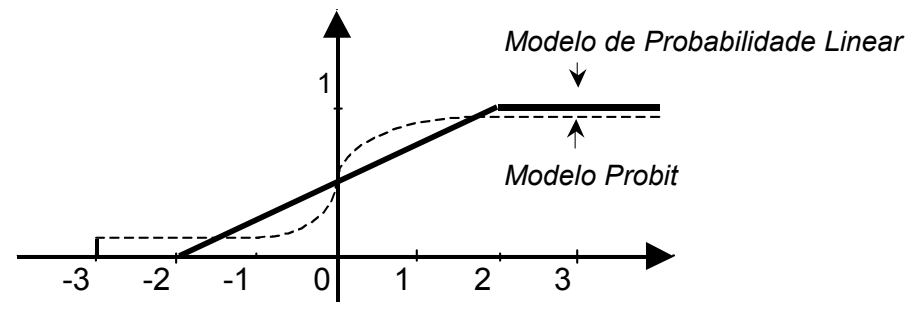

Figura 1. Modelo Probit

\subsection{Dados Utilizados}

Para aplicação das hipóteses foram utilizados dados referentes ao período de 1997 a 2001 de 46 empresas brasileiras que possuem ações negociadas na Bolsa de Valores de São Paulo (BOVESPA). Os dados foram depurados e aplicados nas definições das variáveis de estudo: tamanho, mudança no lucro operacional, retorno das ações, índice $Q$ e a mudança na participação acionária, que podem ser obtidos nos sistemas de informações da Comissão de Valores Mobiliários (CVM), da BOVESPA, nas informações econômico-financeiras da empresa Economática e também mediante informações obtidas dos próprios sites de internet das empresas selecionadas.

Os dados foram analisados em três conjuntos de informação: a) dados relativos ao total da amostra das empresas pelo período total 1997-2000, e também durante cada um dos anos do período, b) 


\section{Revista Eletrônica de Ciência Administrativa (RECADM) - ISSN 1677-7387 \\ Faculdade Cenecista de Campo Largo - Coordenação do Curso de Administração \\ v. 3, n. 1, maio/2004 - http://revistas.facecla.com.br/index.php/recadm/}

dados correspondentes a duas sub-amostras: um grupo de empresas industriais e de manufatura, e outro grupo correspondente às instituições financeiras.

Foram considerados como executivos principais, os cargos de presidente e vice-presidente, assim como os altos cargos de diretoria (Finanças, Produção, Marketing e Recursos Humanos).

Foram considerados como acionistas minoritários àqueles que possuíam menos do $5 \%$ da participação total do conjunto de ações ordinárias.

O método de estimação utilizado é o de painel de dados, na qual observações time- series e crosssection são combinadas e estimadas, ou seja, uma série de observações cross-section são observadas ao longo do período de tempo da pesquisa.

A utilização de painel de dados possui algumas vantagens sobre análises convencionais de crosssection e time-series utilizadas separadamente. Segundo Greene (2000), a utilização de painel de dados torna possível aumentar o número de observações, diminuindo os graus de liberdade e reduzindo a multicolinearidade entre as variáveis explicativas e, desta forma, melhorando a eficiência do modelo econométrico.

Finalmente, com as observações das variáveis analisadas foi executado o modelo Probit Multivariado utilizando-se o software RATS versão 4.0.

\subsection{Metodologia}

As hipóteses a serem testadas serão as seguintes:

Hipótese Básica $\mathbf{H}_{1}$ : Rotatividade do executivo principal está relacionada ao desempenho da companhia (resultados operacionais, valor das ações e valor de mercado).

Hipótese $\mathbf{H}_{2}$ : Rotatividade do executivo principal é insensível às mudanças do acionista controlador.

Hipótese $\mathrm{H}_{3}$ : Rotatividade do executivo está associada ao tamanho da empresa.

Para testar as hipóteses anteriores, aplicamos o modelo a seguir à amostra das 46 empresas selecionadas,

$$
\operatorname{Pr}\left(\text { Rotatividade }_{i t}=1\right)=\Phi\left(\alpha_{t}+\beta_{0} \cdot D_{i t}+\sum_{j=1}^{4} \beta_{j} \cdot \text { Desempenho }_{i t}+\delta \cdot \text { Tamanho }_{i t}+\varepsilon_{i t}\right)
$$


Revista Eletrônica de Ciência Administrativa (RECADM) - ISSN 1677-7387

Faculdade Cenecista de Campo Largo - Coordenação do Curso de Administração

v. 3, n. 1, maio/2004 - http://revistas.facecla.com.br/index.php/recadm/

ou também,

$\operatorname{Pr}\left(\right.$ Rotatividade $\left._{i t}=1\right)=\Phi\left(\alpha_{t}+\beta_{1} \cdot \Delta L O_{i t}+\beta_{2} \cdot T_{i t}+\beta_{3} \cdot R_{i t}+\beta_{4} \cdot Q_{i t}+\beta_{5} \cdot A P_{i t}+\varepsilon_{i t}\right)$

Onde, conforme detalhado na seção 2.2,

$\Delta L O_{i t}$ : Mudança no Lucro Operacional da empresa i no período $t$

$T_{i t}$ : Tamanho da empresa i no período $\mathrm{t}$

$R_{i t}$ : Retorno das ações da empresa i no período $\mathrm{t}$

$Q_{i t}$ : Índice $Q$ de Tobin da empresa i no período $t$

$A P_{i t}$ : Mudança na participação acionária da empresa i no período $t$

A escolha do horizonte de tempo de 1997 a 2000 permite um número suficiente de observações para obter resultados confiáveis. O intervalo de confiança nestes casos pode ser considerado de $95 \%$, ou de $90 \%$, dados os resultados do $\mathrm{R}^{2}$ da regressão Probit.

Em todas as regressões, a matriz de covariância é corrigida para heterocedasticidade conforme White (1980).

\section{Resultados}

Os resultados mostrados na Tabela 1 verificam que quando são analisadas as variáveis para cada ano do período em estudo, unicamente o tamanho da empresa resulta ser significante a um nível de $95 \%$ de confiabilidade.

Os altos valores de $\mathrm{R}^{2}$ determinam um ajuste razoável por parte da modelagem utilizada. Os valores dos coeficientes obtidos para a constante validam a afirmação anterior, aumentando a bondade de ajuste do modelo e visando a diminuição da perturbação aleatória. 
Revista Eletrônica de Ciência Administrativa (RECADM) - ISSN 1677-7387

Faculdade Cenecista de Campo Largo - Coordenação do Curso de Administração

v. 3, n. 1, maio/2004 - http://revistas.facecla.com.br/index.php/recadm/

Tabela 1. Resultados das regressões com modelo Probit da amostra total considerando os anos do período.

\begin{tabular}{|l|c|c|c|c|c|c|c|c|}
\hline \multirow{2}{*}{ Variáveis } & \multicolumn{2}{|c|}{ Amostra 1997 } & \multicolumn{2}{c|}{ Amostra 1998 } & \multicolumn{2}{c|}{ Amostra 1999 } & \multicolumn{2}{c|}{ Amostra 2000 } \\
\cline { 2 - 9 } & Coef. & T-estat. & Coef. & T-estat. & Coef. & T-estat. & Coef. & T-estat. \\
\hline Constante & $-5,55$ & $-1,87$ & $-6,42$ & $-1,98$ & $-5,27$ & $-2,05$ & $-10,52$ & $-2,34$ \\
$\Delta$ Lucro & 14,41 & 1,34 & 6,16 & 0,91 & $-0,20$ & $-0,24$ & 7,52 & 1,04 \\
Op. & 0,41 & 1,98 & 0,51 & 2,18 & 0,32 & 1,99 & 0,68 & 2,44 \\
Tamanho & 0,52 & 0,52 & $-2,80$ & $-1,35$ & 0,85 & 1,55 & $-0,42$ & $-0,23$ \\
Índice Q & $-0,94$ & $-1,34$ & $-0,75$ & $-1,08$ & 0,11 & 0,20 & 0,96 & 0,78 \\
Retorno & 0,46 & 0,80 & $-0,24$ & $-0,28$ & 1,93 & 2,96 & $-0,11$ & $-0,15$ \\
$\Delta$ Part. & & & & & & & & \\
Acion. & & & & & & & & \\
\hline $\mathbf{R}^{2}$ & 0,92 & & 0,85 & & 0,89 & & 0,91 & \\
\hline \hline
\end{tabular}

Como pode ser observado, existiria uma relação de causalidade entre o tamanho do negócio e a rotatividade, como função do aumento no nível de responsabilidade gerencial. É interessante observar que as outras variáveis são estatisticamente não significantes.

A tabela 2 apresenta resultados utilizando um maior numero de observações, ou seja, todo o horizonte de análise. Os resultados em relação ao coeficiente da constante e da variável tamanho da empresa são significantes a um nível de $90 \%$ de significância estatística para os três grupos. Em relação à causalidade da variável mudança na participação acionária, os resultados mostraram-se significantes para o total da amostra e para o grupo de empresas industriais, mantendo-se não significante para o grupo das instituições financeiras. 
Revista Eletrônica de Ciência Administrativa (RECADM) - ISSN 1677-7387

Faculdade Cenecista de Campo Largo - Coordenação do Curso de Administração

v. 3, n. 1, maio/2004 - http://revistas.facecla.com.br/index.php/recadm/

Tabela 2. Resultados das regressões do modelo Probit da amostra total, considerando o período 1997-2000.

\begin{tabular}{|c|c|c|c|c|c|c|}
\hline \multirow[t]{2}{*}{ Variáveis } & \multicolumn{2}{|c|}{$\begin{array}{c}\text { Total } \\
1997-2000\end{array}$} & \multicolumn{2}{|c|}{$\begin{array}{l}\text { Indústrias } \\
1997-2000\end{array}$} & \multicolumn{2}{|c|}{$\begin{array}{c}\text { Inst. Financeiras } \\
1997-2000\end{array}$} \\
\hline & Coef. & T-estat. & Coef. & $T$-estat. & Coef. & T-estat. \\
\hline Constante & $-3,98$ & $-3,38$ & $\begin{array}{l}-0,36 \\
\end{array}$ & $-2,19$ & 16,92 & 0,75 \\
\hline$\Delta$ Lucro Op. & 1,06 & 0,55 & $-0,21$ & $-0,09$ & 24,75 & 1,19 \\
\hline Tamanho & 0,28 & 3,52 & 0,44 & 1,69 & $-1,14$ & $-1,85$ \\
\hline Índice Q & 0,13 & 0,38 & 0,49 & 1,29 & 14,69 & 0,80 \\
\hline Retorno & $-0,23$ & $-1,12$ & $-0,13$ & $-0,60$ & $-0,58$ & $-0,34$ \\
\hline $\begin{array}{l}\Delta \text { Part. } \\
\text { Acion. }\end{array}$ & 0,54 & 2,06 & 0,35 & 1,98 & 5,23 & 0,79 \\
\hline$\overline{\mathbf{R}^{2}}$ & 0,82 & & 0,80 & & 0,76 & \\
\hline
\end{tabular}

Os valores de $\mathrm{R}^{2}$, ao igual que o caso anterior, determina um ajuste razoável por parte do modelo utilizado.

\section{Conclusões}

Os resultados deste trabalho mostram que é possível encontrar mecanismos de avaliação dos sistemas de governança corporativa das empresas. A confiabilidade dos resultados dependerá em grande parte da quantidade e qualidade das informações que possam ser coletadas para cumprir com a finalidade específica do tipo de análise a ser realizado. Nesse sentido, foi possível examinar a eficiência da governança das empresas através da rotatividade dos executivos. Assim, utilizaram-se os modelos Probit, que apresentaram alguns resultados significantes.

Em relação à Hipótese 1, os resultados mostraram que, com exceção da variável tamanho da empresa, as outras variáveis mostraram-se estatisticamente não significantes. Isto significa que variáveis de performance e valor da firma parecem não ter incidência nas mudanças da variável dependente (rotatividade empresarial).

Em relação à Hipótese 2, os resultados apresentados foram inconclusivos para a relação entre a variável dependente e as mudanças na participação dos acionistas.

Em relação à Hipótese 3 , existe uma relativa associação revelada pela baixa significância entre a variável rotatividade executiva das empresas e a variável definida pelo tamanho da empresa. 
Revista Eletrônica de Ciência Administrativa (RECADM) - ISSN 1677-7387

Faculdade Cenecista de Campo Largo - Coordenação do Curso de Administração

v. 3, n. 1, maio/2004 - http://revistas.facecla.com.br/index.php/recadm/

\section{REFERÊNCIAS BIBLIOGRÁFICAS}

ALDRED, C. U.K Risk Managers`stock May Be Rising. Business Insurance, Chicago, 4 de junho de 2001.

ANDERSON, Ronald C. Corporate governance and Firm Diversification. Financial Management, spring 2000.

BEBCHUK, L. A rent-protection theory of corporate ownership and control. NBER Working Paper, $n$. 7203, 1999.

Business Europe`s Revolting Shareholders - The Economist, London, 12 de maio de 2001.

COFFEE, J. The future as history : the prospects for global convergence in corporate governance and its implications. Northwestern University Law Review, n. 93, p. 641-708, 1999.

Experiências de Governança Corporativa. Harvard Business Review, São Paulo: Campus, 2001.

FRANKS, J.; MAYER, C. Ownership and Control of German Corporations. Manuscrito do London School of Economics, UK, 2000.

Instituto Brasileiro Governança Corporativa - <http://www.ibgc.com.br>

GOMPERS, P.; ISHII J.; METRICK A. Corporate Governance and Equity Prices. NBER Working Paper No. w8449. August 2001.

GREENE, William H. Econometric Analysis, $4^{\text {th }}$ Ed, Prentice Hall, 2000.

KAPLAN, S. Top executive rewards and firm performance: a comparison of Japan and the United States. Journal of Political Economy, n. 120, p. 510-546, 1994.

KAPLAN, S. Top executives, turnover, and firm performance in Germany. Journal of Law, Economics \& Organization, n. 10, p.142-159, 1994.

LA PORTA, R.; LOPEZ-DE-SILANES, F.; SHLEIFER, A. Corporate ownership around the world. Journal of Finance, n. 54, p. 471-517, 1999. 
Revista Eletrônica de Ciência Administrativa (RECADM) - ISSN 1677-7387

Faculdade Cenecista de Campo Largo - Coordenação do Curso de Administração

v. 3, n. 1, maio/2004 - http://revistas.facecla.com.br/index.php/recadm/

LODI, João Bosco. Governança Corporativa, o Governo da Empresa e o Conselho de Administração. São Paulo: Campus, 2000.

MARSHALL, J. As Boards Shrink,Responsibilites Grow. Financial Executive, jun. 2001.

MADDALA, G. S. Introduction to Econometrics. New York: Macmillan Publishing Company, 1988

McCONNELL, J.; Servaes, H. Additional evidence on equity ownership and corporate value. Journal of Financial Economics , n.26, p. 595-612, 1990.

MORCK, R., Shleifer, A., Vishny, R. Management ownership and market valuation. Journal of Financial Economics, n. 20, p. 293-315, 1988.

PINDYCK, Roberts; RUBINFELD, Daniel L. Econometric Models and Time Series. Mc Graw-Hill: New york, 1986.

VOLPIN, Paolo F. Governance with poor investor protection: evidence from top executive turnover in Italy. Jounal of Financial Economics Manuscript, Set. 2001.

WARNER, J.; WATTS, R.; WRUCK, K. Stock prices, event prediction and event studies: An examination of top management restructuring. Journal of Financial Economics 20, p.461-492, 1988.

WHITE, Halbert. A heteroskedasticity-consistent covariance matrix estimator and direct test for heteroskedasticity. Econometrica, 1980, Vol. 48, 817-838. 